\title{
CDISC SEND Study Type Terminology
}

National Cancer Institute

\section{Source}

National Cancer Institute. CDISC SEND Study Type Terminology. NCI Thesaurus. Code C90003.

The terminology that includes concepts relevant to the Clinical Data Interchange Standards Consortium (CDISC) Standard for the Exchange of Non-clinical Data (SEND) study types. 